\title{
Lifetest of the High Output Maximum Efficiency Resonator (HOMER) Laser for the SAFFIRE Instrument on NASA's DESDynI Project
}

\author{
Paul R. Stysley*, D. Barry Coyle*, Richard B. Kay**, Robert Frederickson $\dagger$, Demetrios Poulios**, Bryan \\ Blair*, Stan Scott*, and Ed Arnold $\dagger$ \\ *NASA/Goddard Space Flight Center, Greenbelt, MD 20771 \\ paul.stysley@nasa.gov \\ **Department of Physics, American University, Washington, DC 20016 \\ †S Science Systems Applications Inc., Lanham MD
}

\begin{abstract}
We update the status of a diode-pumped, Nd:YAG oscillator that is the prototype laser for NASA's DESDynI mission. After completing TRL-6 testing, this laser has fired over 5.5 billion shots in lifetesting. (c)2011 Optical Society of America

OCIS codes: $140.3480,140.3540,140.3580,280.3640$
\end{abstract}

The HOMER (High Output Maximum Efficiency Resonator) laser is to be deployed on the SAFFIRE (Spaceborne Altimeter For Forest \& Ice Research and Exploration) instrument on NASA's DESDynI (Deformation, Ecosystem Structure and Dynamics of Ice) Earth science program. At the time of publication, DESDynI is designed to employ, along with a SAR, a multi-beam LIDAR in a $374 \mathrm{~km}$ sun synchronous orbit, producing five $1064 \mathrm{~nm}$ beams with 25 $\mathrm{m}$ footprints on the Earth. DESDynI's LIDARs will study the Earth's biomass and glacial ice for a minimum of 2 years [1]. The SAFFIRE instrument requires that each laser fire at 241 pulses per second, produce a 9-10 ns laser pulsewidth, and project a $17 \mathrm{~mJ}$ pulse with a TEM0o beam quality. This mission needs a highly efficient and reliable laser instrument that will be capable of in excess of 6 billion shots over the lifetime of the mission, with a goal of 10 billion shots for margin. HOMER has already successfully met the beam output requirements in previous studies and experiments, firing over 6 billion shots at DESDynI mission relevant settings [2]. To further increase confidence in the design, HOMER was mechanically upgraded from its earlier EDU configurations so that it could effectively complete space-worthy TRL-6 (Technology Readiness Level 6) testing, including thermal vacuum (TVAC) tests, vibration tests, and laser life testing. This new version was designated HOMER-2, as shown in figure 1. It is important to note that through all of this testing and experimentation we found the fundamentals and advantages of an oscillator-only design over a MOPA system remain the same. HOMER remains a single actively Q-switched cavity with an $808 \mathrm{~nm}$ side pumped Nd:YAG gain medium employing a positive branch unstable resonator (PBUR) [3]. The TVAC testing confirmed HOMER's survival temperature range of $0-40 \mathrm{C}$ specification by performing eight thermal cycles ranging from $50 \mathrm{C}$ to $-10 \mathrm{C}$ with 4 hour dwell times. TRL 6 testing also involved vibration testing at the General Environmental Verification Standard (GEVS) in the $\mathrm{x}, \mathrm{y}$, and $\mathrm{z}$ axises. Vibration testing exposed subtle flaws in the mechanical interfaces between the gain module and optical bench, as well as the

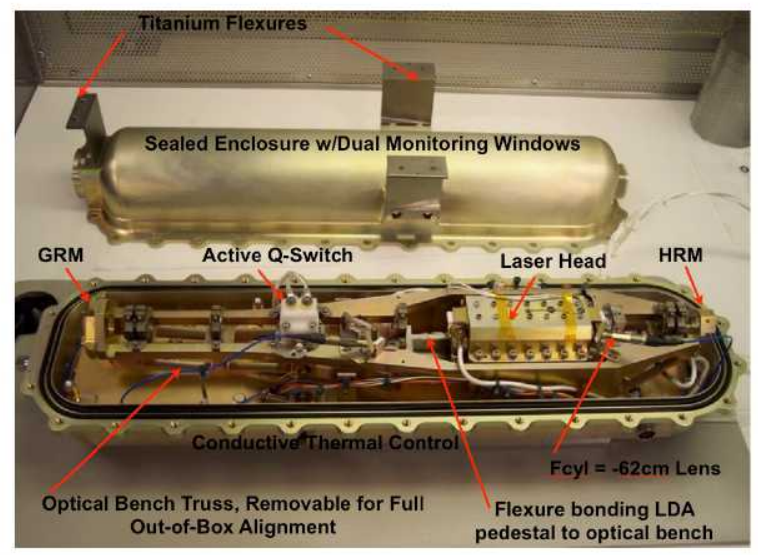

Figure 1. The fully assembled HOMER-2 laser with the enclosure open for pre-lifetest inspection. Note, the flexures are mounted to the "lid" and the radiator panel interface for HOMER-2 is below.

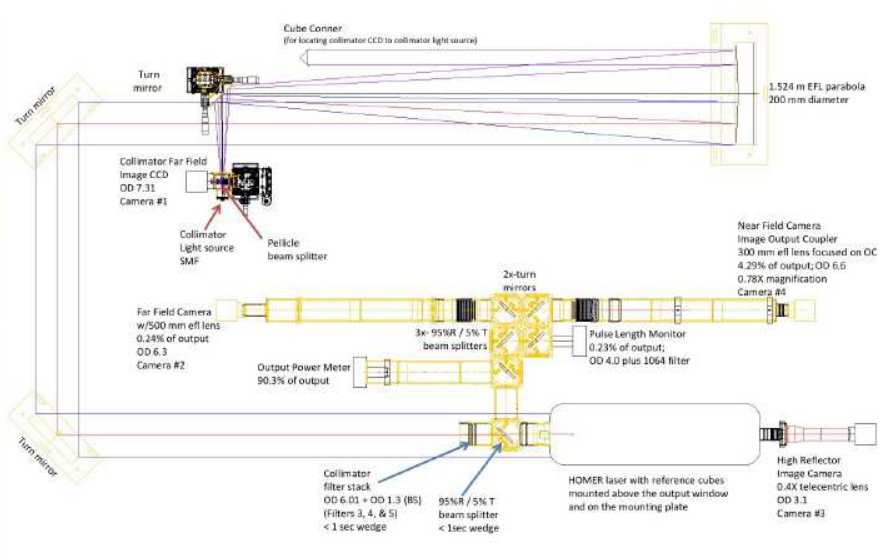

Figure 2. Optical layout of the HOMER-2 lifetest. A 1064nm fiber coupled laser diode is reflected off reference cubes mounted on the enclosure and the base plate. These determine any movement of laser cavity vs. the outside environment. 
housing's titanium flexures. Quick modifications were made to validate the team's theories, and repeated vibration tests showed immediately that these were the correct areas to focus on in the coming HOMER-ETU effort [4].

HOMER's optics were then inspected to confirm that no optical damage or degradation occurred. Next we proceeded to do a final torquing, enclosure seal, and system integration for lifetime testing. The lifetest is currently underway in a non-cleanroom laser lab environment at DESDynI-relevant parameters. We plan on operating at $\geq 15$ $\mathrm{mJ}$ pulses and $241 \mathrm{~Hz}$ for over a year until 10 billion shots are achieved. The lifetime experimental setup can be seen in figure 2. To maximize the lifetest operation and minimize the degradation rate, the LDA's are driven far below the manufacturer's rated limits. Thus we have shown a very low measured degradation rate of $\sim 250 \mathrm{uJ} / \mathrm{B}$ shots, which we readily attribute to the $51 \%$ current derating and low pump pulse width of 65 us, for a low duty cycle of $1.5 \%$. Furthermore, should the laser catastrophically drop bars in the mission, there is more than enough range in the drive electronics and LDA specifications to turn up the current and/or pulsewidth to compensate. The $\mathrm{Nd}$ :YAG slab is chromium co-doped for radiation hardening and, pumped by seven, 4-bar G-package laser diode arrays, and rated at $100 \mathrm{~W} / \mathrm{bar}$ at $100 \mathrm{~A}$. At beginning of life (BOL), HOMER-2 emitted as high as $15.7 \mathrm{~mJ}$, with a far field divergence of $1.0 \mathrm{mR} \times 0.9 \mathrm{mR}$ shown in figure 3 . After $3 \mathrm{~B}$ shots, the pulse energy dropped to $15 \mathrm{~mJ}$ at which point we turned up the current by 1 A giving an output energy of $16 \mathrm{~mJ}$. As of the date of publication, the laser has achieved over 5.5 billion shots and is currently running at $15.5 \mathrm{~mJ}$. It has experienced no more than a 0.25 $\mathrm{mJ} / \mathrm{B}$ energy degradation over time as seen in figure 4 .

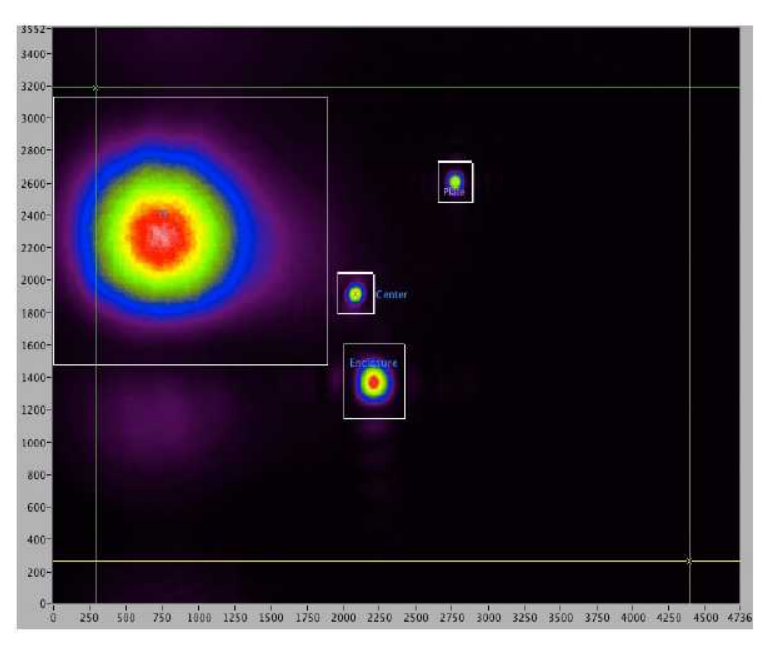

Figure 3. The CCD image simultaneously displays all reference beams and the HOMER-2 far field beam. The far field measures approximately $0.9 \mathrm{mR} \times 1.0 \mathrm{mR}$.

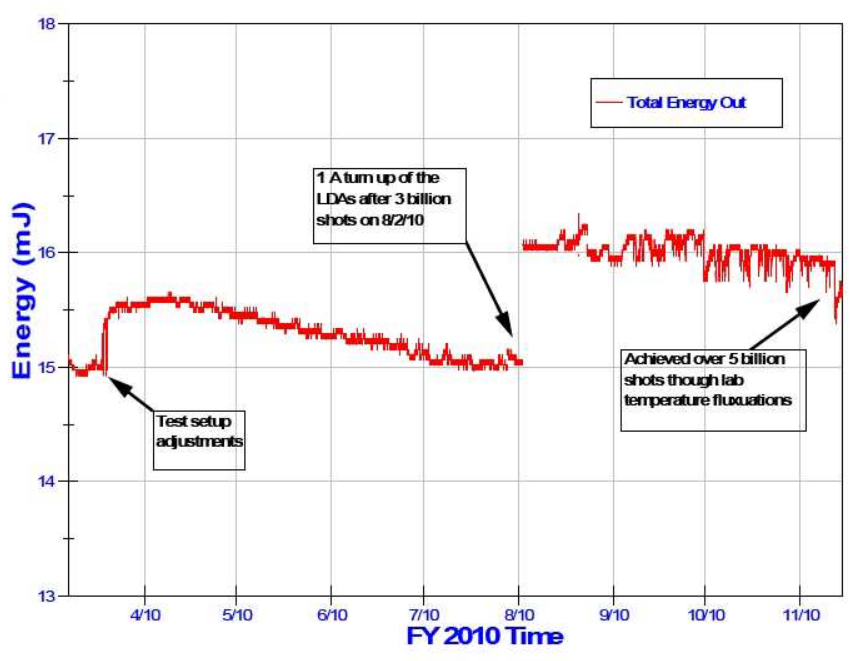

Figure 4. Summary of the over 5.5 billion shots fired by HOMER during its 9 months of lifetesting. HOMER has experience no more than $0.25 \mathrm{~mJ} / \mathrm{B}$ loss between milestones.

In the upcoming year we plan to build another mechanical upgrade to the laser system for the DESDynI program. This HOMER-ETU will apply lessons learned from the above reported TRL- 6 environmental and lifetime testing. It will also incorporate a flight prototype beam expander, LDA drive electronics, and a flight-like laser control unit. These upgrades combined with HOMER will be considered the SAFFIRE laser instrument and will go through more rigorous DESDynI LIDAR relevant TRL-6 level testing.

\section{References}

[1] http://desdyni.jpl.nasa.gov

[2] D. Barry Coyle and Paul Stysley, "The High Output Maximum Efficiency Resonator (HOMER) Develop for Long Life, Space Based Vegetation and Surface Imaging” Aerospace conference, IEEE, 2006.

[3] D. Barry Coyle, Richard B. Kay, Paul R. Stysley, Demetrios Poulios, "Efficient, Reliable, Long-lifetime, Diode-pumped Nd:YAG Laser for Space-based Vegetation Topographical Altimetry,” App. Opt. 43, 5236-5242 (2004).

[4] D. Barry Coyle, Paul R. Stysley, Peter Rossoni, Robert Frederickson, Cheryl Salerno, Richard B. Kay, Demetrios Poulios, Bryan Blair, and Ken Cory, "Flight Qualification of the High Output Maximum Efficiency Resonator (HOMER) Laser for Space-based Remote Sensing Applications" CLEO Conference, 2009. 